\title{
An Iterative Synthetic Aperture Imaging Algorithm with Correction of Diffraction Effects
}

\author{
Erik Wennerström, Tadeusz Stepinski, Senior Member, IEEE, and Tomas Olofsson
}

\begin{abstract}
In this paper we present an iterative version of the synthetic aperture imaging algorithm extended synthetic aperture technique (ESAFT) proposed recently. The algorithm is based on a linear model that accounts for the distortions effects of an imaging system used for acquisition of ultrasonic data. Improved resolution (both lateral and temporal) in the reconstructed image is obtained as a result of minimizing the reconstruction mean square error. In this work, the minimization is extended to parameters that characterize expected amplitudes of each image element in the area of interest. An iterative optimization scheme is proposed, which in each step performs minimization of the reconstruction error based on the parameter matrix found in the previous step. Comparing to ESAFT, the proposed approach yields a significant improvement in resolution and a high degree of robustness with regard to initial choice of the parameter matrix. Performance of the proposed algorithm is evaluated using both real and simulated ultrasonic data.
\end{abstract}

\section{INTRODUCTION}

Synthetic aperture imaging (SAI) includes a number Sof techniques aimed at improving the lateral resolution of ultrasound images by extending aperture of a physical source achieved by processing several successive measurements. The primary SAI technique, known as synthetic aperture focusing technique (SAFT) [1] has been inspired by synthetic aperture sonar (SAS) [2] and synthetic aperture radar (SAR) [3]. SAFT has been applied both in medical and nondestructive material evaluation (NDE) applications. All SAI algorithms include various postprocessing techniques used for obtaining all-point dynamic focusing with a uniform resolution based on multiple consecutive illuminations from different points located on a line.

Classical algorithms used for SAI are based on the implicit assumption, which yields both in SAR and SAS that the diffraction effects of the transducers can be neglected. However, in practical applications, the lateral resolution of many SAI algorithms is often limited by the non-negligible size of the transducer used for data acquisition [4] [5]. The minimum transducer size in ultrasound can be limited by, for instance, the minimum area needed for obtaining the desired electric characteristics, or the minimum transmitted acoustical power required for obtaining a sufficient signal-to-noise ratio (SNR).

Manuscript received April 22, 2005; accepted December 21, 2005. The authors are with Uppsala University, Signals and Systems group, Uppsala, Sweden (e-mail: ew@signal.uu.se).
An attempt to overcome the problems resulting from a finite-active area of the transducer, proposed in [6] was using a relatively large focused aperture (virtual source). In this method, a virtual source that emits a spherical wave is assumed to be located in the focal point of the aperture. The virtual source technique has been investigated by a number of researchers [7]-[9]; however, in in some cases it has not yielded the initially anticipated improvements [8].

A more straightforward approach consists in taking into account the spatial characteristics of the transducer when developing the SAI algorithm. A time-domain solution to this problem was presented recently under the name ESAFT [10] [11]. ESAFT is capable of compensating the diffraction effects of a finite-sized transducer, both in transmission and reception. It has been shown that this technique is superior to many other common SAI algorithms, such as conventional SAFT and phased-array techniques concerning lateral resolution [12], [13].

In ESAFT, which is inspired by the estimation theory of linear systems, the imaging problem is formulated as the estimation of a set of reflectivity amplitudes defining a so-called object function, see Section II. ESAFT is the linear solution to this problem, optimal in the mean-squared error (MSE) sense. This model is especially useful in NDE that in most cases only a limited number of strong scatterers is encountered in a homogenous medium. ESAFT, which can be interpreted as a spatiotemporal filter, is defined in terms of the spatial impulse responses (SIRs) of the transducers used for imaging. It further requires knowledge about the covariance matrix of the measurement errors as well as the covariance matrix describing the prior uncertainty about the elements in the object function.

The choice of covariance matrix for the measurement errors seldom causes any serious problem as the measurement noise typically can be well modeled as zero mean, independent, and identically distributed Gaussian sequence. Thus, the noise components share a single scalar variance that usually can be estimated from data.

However, choosing the covariance matrix for the elements in the object function in the region of interest (ROI) presents a challenge. Moreover, this choice may have a large influence on quality of the resulting image. A conservative choice consists in setting this matrix equal to the identity matrix multiplied by a scalar variance. This model represents an equal uncertainty about all amplitudes in the object function. In practice, the scalar variance usually is seen as a tuning parameter used by an operator to 
trade between image resolution and noise level. Setting the variance to a small value has the drawback that large amplitudes in the image are underestimated, and the energy is smeared over a neighboring area around such a point. Setting it to large values improves the resolution, but at the cost of an increased noise level.

In many NDE applications, the inspected object contains only a finite number of strong reflectors whereas most of the points in the inspected image plane reflect little energy back. A typical example is NDE of welded metal structures in which cavities and cracks are to be detected. In medical ultrasonic applications, the images usually are less sparse and have less contrast, as the medium is less homogenous.

If the above mentioned conservative choice of covariance matrix is to be made in such varying conditions, the operator using ESAFT may need to spend much time to find the parameter resulting in an optimal trade-off between resolution and noise level. It is important to note that this trade-off is mainly a result of the choice of the overly simple structure of the covariance matrix. Associating individual variances with all amplitudes in the object function provides means for controlling the resolution and noise amplification properties in the reconstruction.

In this paper, a further refinement of the ESAFT technique aimed at enhancing lateral resolution and reducing background noise in the processed image is proposed. This is achieved by a simple adaptation scheme of the elements in the covariance matrix describing the reflectivity amplitudes. The proposed algorithm is a two-dimensional (2-D) extension of a 1-D method proposed in [14]. The method takes the form of an iterative scheme and is shown to offer an improvement over the standard ESAFT algorithm both in terms of resolution and background noise level. The parameter setting also is simplified compared to ESAFT in the sense that the results are less sensitive to the small variations in the user parameters.

The reminder of this paper is organized as follows. In Section II, the discrete model of the imaging system is presented. The solution to the inverse problem is presented in Section III in which also the algorithm is derived. To illustrate the resolution of the proposed iterative scheme, Section IV presents numerical results obtained from simulations and measurements. Conclusions are presented in Section V.

\section{Discrete Linear Model of the Imaging System}

ESAFT presented in [10] and the iterative ESAFT, presented in this paper, are both model-based statistical approaches to ultrasonic synthetic-aperture imaging. The model of the imaging system is linear and discrete and includes diffraction effects introduced by the ultrasonic transducer. The presence of additive noise is assumed in the model, and a reconstruction filter is designed to yield the best estimate of the original image. In this section, the

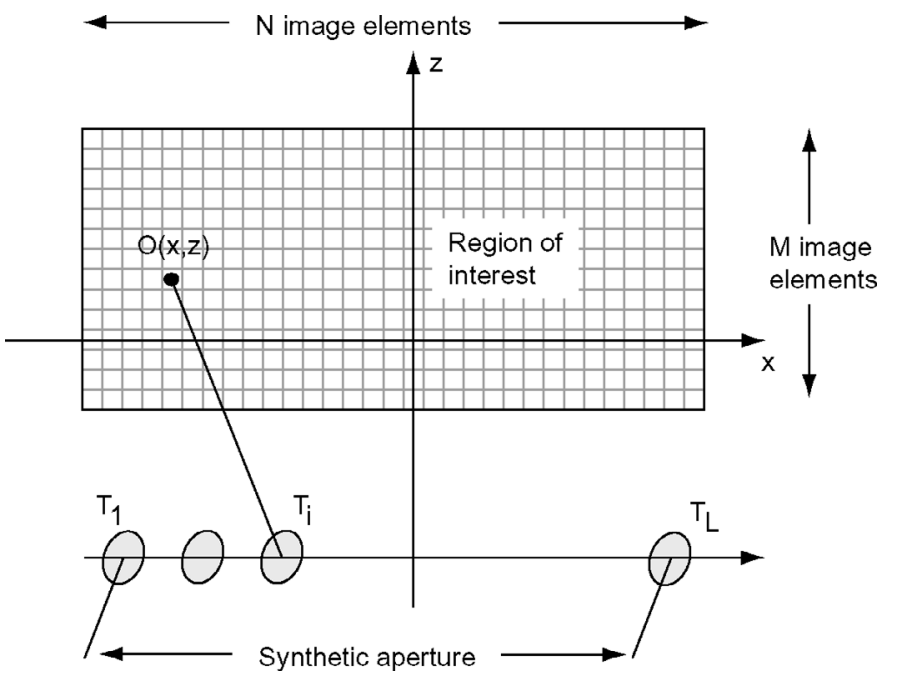

Fig. 1. Monostatic synthetic aperture geometry. In each position $T_{1}, T_{2}, \ldots, T_{L}$ the transducer emits a pulse and receives an echo from a scatterer located in point $(x, z)$.

linear model of the imaging system is shortly outlined. A more detailed presentation can be found in [10].

Consider the measurement setup shown in Fig. 1 in which the ROI that is imaged is denoted by $\mathbf{O}$. The ROI that is divided into $M \times N$ image elements is located in the $x z$-plane with $y=0$. Every element $o(x, z)$ in the image $\mathbf{O}$ is a scalar representing the scattering strength of a target in the position $(x, z)$. Measurements from $L$ positions of a transducer moving parallel to the $\mathrm{x}$-axis are available in discrete-time form. The measurement set consists of $L$ A-scans, each containing $K$ samples.

Such a setup in which both transmission and reception is performed with a single transducer is denoted as a monostatic synthetic aperture. This is the setup considered throughout this paper. Note, however, that it is possible to transmit with a single transducer and to receive with separate transducers in all other measurement points $T_{l}$. In this multistatic acquisition scheme, the use of several transducers provides more information about the ROI. The extra data could be incorporated into this model and, with the added information, there might be an improvement in the results, at a cost of increased computation effort.

Let the $K \times 1$ vector $\mathbf{y}_{l}$ denote the noise-free A-scan response at position $T_{l}$, which can be expressed as a sum of echoes from all scatterers in the ROI. Each echo can be modeled as a convolution between an electro-mechanical impulse response and a double-path SIR [13]. The double path SIR defines the relation between the particle velocity at the transducer surface at transmission, and the instantaneous pressure at the transducer surface in reception. The electromechanical impulse response models the combined electrical-to-acoustical and acoustical-toelectrical properties of the transducer both in transmission and reception. Let $\mathbf{p}_{l}(x, z)$ denote the $K \times 1$ vector that consist of the combined time discreet electrical and spatial impulse response of a scatterer at position $(x, z)$ and a transducer at position $l$. Calculating the SIRs of a trans- 
ducer naturally requires information about its geometry and focusing, but even for complicated geometries the SIRs can be calculated using numerical methods.

By neglecting multiple scattering effects, an A-scan can be modeled as a superposition of the contributions from all scatterers in the ROI. Using the notation developed above, the A-scan can be written as:

$$
\mathbf{y}_{l}=\sum_{n=0}^{N-1} \sum_{m=0}^{M-1} \mathbf{p}_{l}\left(x_{n}, z_{m}\right) o\left(x_{n}, z_{m}\right) .
$$

The $L$ vectors $\mathbf{y}_{0}, \ldots, \mathbf{y}_{L-1}$ now can be stacked on each other to form a $K L \times 1$ vector, $\mathbf{y}$. In a similar way, a $M N \times 1$ vector, $\mathbf{o}$ is formed by stacking all elements in $\mathbf{O}$. Form the size $K \times M$ matrices:

$$
\mathbf{P}_{l}(n)=\left(\mathbf{p}_{l}(n, 1) \ldots \mathbf{p}_{l}(n, M)\right),
$$

for all combinations of transducer positions $l$ and columns in $\mathbf{O}$. Now the SIR matrix $\mathbf{P}$ can be constructed as:

$$
\mathbf{P}=\left(\begin{array}{cccc}
\mathbf{P}_{0}(0) & \mathbf{P}_{0}(1) & \ldots & \mathbf{P}_{0}(N-1) \\
\mathbf{P}_{1}(0) & \mathbf{P}_{1}(1) & \ldots & \mathbf{P}_{1}(N-1) \\
\vdots & & & \vdots \\
\mathbf{P}_{L-1}(0) & \mathbf{P}_{L-1}(1) & \ldots & \mathbf{P}_{L-1}(N-1)
\end{array}\right)
$$

The matrix $\mathbf{P}$ contains all the combined electrical and spatial impulse responses for all combinations of points $(x, z)$ in the image and all transducer positions $l$. Using this matrix, it is possible to write the model in the compact notation:

$$
\mathbf{y}=\mathbf{P o}+\mathbf{e}
$$

where $\mathbf{e}$ is additive measurement noise. See [13] and [15] for details concerning the impulse responses, the structure of $\mathbf{P}$, and other details of this model.

\section{The Inverse Filter, Minimization Problem}

The ESAFT algorithm is based on the linear convolution model of the imaging system defined in (4). The approach proposed in [10] consists in finding $\hat{\mathbf{o}}$ from $\mathbf{y}$ using the reconstruction filter $\mathbf{K}$ that minimizes the mean square error $J_{M M S E}=E\left\{\|\mathbf{o}-\mathbf{K} \mathbf{y}\|^{2}\right\}$.

This is equivalent to finding the maximum a postiori (MAP) estimate of $\mathbf{o}$, assuming that $\mathbf{o}$ and $\mathbf{e}$ are Gaussian [13]. The MAP loss function can be written as:

$$
\begin{aligned}
& \underset{\mathbf{o}}{\arg \max } p(\mathbf{o} \mid \mathbf{y})= \\
& \underset{\mathbf{o}}{\arg \max }\left\{\frac{1}{2}(\mathbf{y}-\mathbf{P o})^{T} \mathbf{C}_{\mathbf{e e}}{ }^{-1}(\mathbf{y}-\mathbf{P o})+\frac{1}{2} \mathbf{o}^{T} \mathbf{C}_{\mathbf{o o}}{ }^{-1} \mathbf{o}\right\}= \\
& \underset{\mathbf{o}}{\arg \max } J .
\end{aligned}
$$

The MAP estimate that minimizes $J$ can be expressed in a closed form:

$$
\hat{\mathbf{o}}=\left(\mathbf{C}_{\mathbf{o o}}{ }^{-1}+\mathbf{P}^{T} \mathbf{C}_{\mathbf{e e}}{ }^{-1} \mathbf{P}\right)^{-1} \mathbf{P}^{T} \mathbf{C}_{\mathbf{e e}}{ }^{-1} \mathbf{y},
$$

where $\mathbf{C}_{\mathbf{o o}}$ is the $M N \times M N$ covariance matrix of the image vector $\mathbf{o}$ and $\mathbf{C}_{\mathbf{e e}}$ is the $K L \times K L$ covariance matrix of the noise $\mathbf{e}$. Under the assumption that the noise $\mathbf{e}$ is Gaussian and white with variance $\sigma_{e}^{2}$, the expression (6) can be simplified to:

$$
\hat{\mathbf{o}}=\left(\mathbf{C}+\mathbf{P}^{T} \mathbf{P}\right)^{-1} \mathbf{P}^{T} \mathbf{y}
$$

where:

$$
\mathbf{C}=\sigma_{e}^{2} \mathbf{C}_{\mathbf{o o}}{ }^{-1} .
$$

If no assumptions that intensities of different elements in the image are correlated to each other are made, $\mathbf{C}_{\mathbf{o o}}$ becomes diagonal and its inverse also is diagonal. $\mathbf{C}$ can now be written as:

$$
\mathbf{C}=\sigma_{e}^{2} \mathbf{C}_{\mathbf{o o}}{ }^{-1}=\left(\begin{array}{cccc}
\delta_{1}^{2} & 0 & \ldots & 0 \\
0 & \ddots & & \\
\vdots & & & \\
0 & & & \delta_{M N}^{2}
\end{array}\right),
$$

where:

$$
\delta_{i}^{2}=\frac{\sigma_{e}^{2}}{\sigma_{o_{i}}^{2}}
$$

and $\sigma_{o_{i}}^{2}$ is the i:th diagonal element of $\mathbf{C}_{\mathbf{o o}}$. The inverse of an element $\delta_{i}^{2}$ on the diagonal of $\mathbf{C}$ describes how great an amplitude of $o_{i}$ can be expected. In the standard ESAFT algorithm, all $\delta_{i}$ are set to the same value. If they are set to large values, high amplitudes are punished, thus suppressing noise. If they are set close to zero, higher amplitudes are allowed, but at the price of increased noise. Should it be possible to have low values on the $\delta_{i}$ in which scatterers are located in the image and high values everywhere else, low noise and good amplitude resolution could be combined.

The main idea of the extension to ESAFT proposed here is to achieve such effect by making $\mathbf{C}$ adaptive and updating the values on the diagonal in several steps. Some constraints are needed to keep the various $\delta_{i}$ from growing too large or coming too close to zero. More on the formulation of the constraints later [see (15) and (16)].

Now, consider the minimization of $J$ with respect to both $\mathbf{o}$ and $\mathbf{C}$. It can be done in alternating steps, first find:

$$
\hat{\mathbf{o}}_{1}=\left.\underset{\mathbf{o}}{\arg \min } J\right|_{\mathbf{C}=\lambda_{0} \mathbf{I}},
$$

using the result in (7). $\mathbf{C}$ is in this step set to the identity matrix multiplied by a constant, $\lambda_{0}$. Note that $\lambda_{0}$ has a physical meaning as the quotient between the variance of the measurement noise in the data $\mathbf{y}$ and the average variance of the elements in $\mathbf{o}$.

Then minimize $J$ again with respect to $\mathbf{C}$ :

$$
\mathbf{C}_{1}=\left.\underset{\mathbf{C}}{\arg \min } J\right|_{\mathbf{o}=\hat{\mathbf{o}}_{1}},
$$



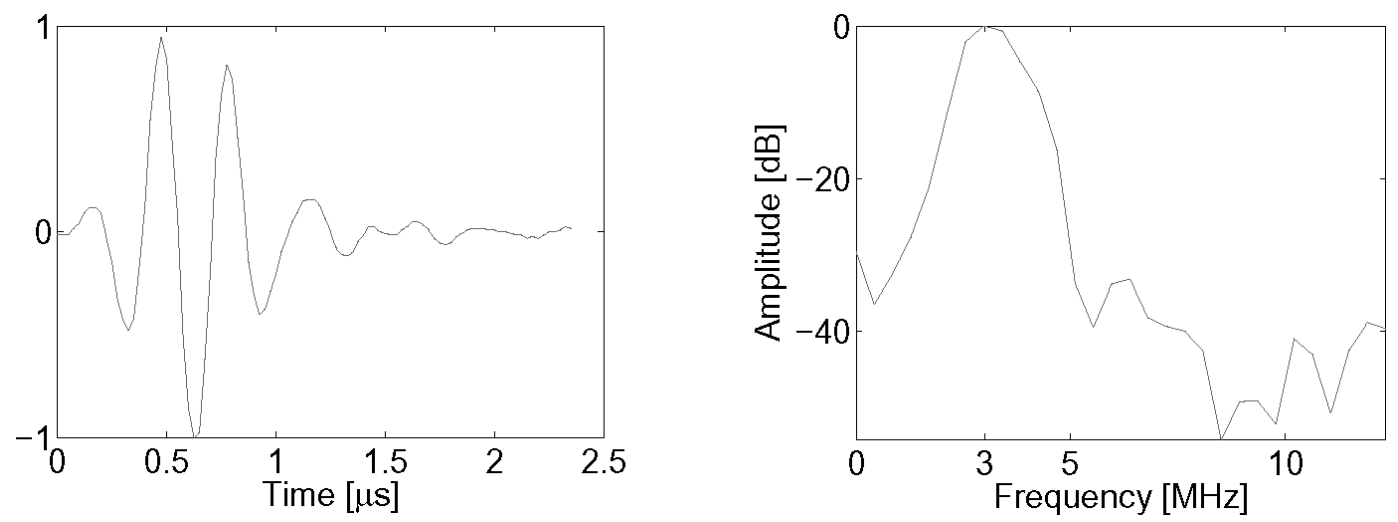

Fig. 2. Impulse response of the transducer (left) and its Fourier transform (right).

that is, when $\mathbf{o}=\hat{\mathbf{o}}$, find the $\mathbf{C}$ that minimizes $J$. The resulting update of $\mathbf{C}$ then is used to find a new estimate of $\mathbf{o}$, and so forth. This process is repeated until convergence.

The algorithm can be summarized in five steps:

- Initialize $k:=1$ and $\mathbf{C}_{0}=\lambda_{0} \mathbf{I}$.

- Find the $k$ :th estimate of the image, using the $k-1$ :st update of the matrix $\mathbf{C}$ :

$$
\hat{\mathbf{o}}_{k}=\left.\underset{\mathbf{o}}{\arg \min } J\right|_{\mathbf{C}=\mathbf{C}_{k-1}} .
$$

- Find the $k$ :th update of $\mathbf{C}$, using the estimate of the image from the last step:

$$
\mathbf{C}_{k}=\left.\underset{\mathbf{C}}{\arg \min } J\right|_{\mathbf{o}=\hat{\mathbf{o}}_{k}} .
$$

- Set $k:=k+1$.

- Repeat step 2, 3, and 4 until $J$ converges.

To eliminate trivial solutions when minimizing $J$ in (13) and (14), constraints have to be imposed on $\mathbf{C}$. Without such constraints, various diagonal elements $\delta_{i}$ in $\mathbf{C}$ could quickly grow out of proportion or quickly diminish. Please recall that $M N$ is the number of elements in the image and consider the constraints:

$$
\begin{aligned}
& \frac{1}{M N} \sum_{i} \frac{\sigma_{o_{i}}^{2}}{\sigma_{e}^{2}}=\frac{1}{M N} \sum_{i} \frac{1}{\delta_{i}^{2}}=\lambda_{0}, \\
& \frac{1}{M N} \sum_{i} \frac{\sigma_{e}^{2}}{\sigma_{o_{i}}^{2}}=\frac{1}{M N} \sum_{i} \delta_{i}^{2}=A .
\end{aligned}
$$

The first constraint (15) roughly expresses the condition that the average energy in the image should not change. Initial assessments of the measurement noise and the image energy are made when minimizing $J$ in (13), and those should hold in each step of the iterated minimization.

The second constraint takes the form of a lower limit for $\sigma_{o_{i}}>0$; indeed, if $\sigma_{o_{i}}$ tended to 0 it would be equivalent to assuming that there is nothing in the corresponding area in the image. This would not be a sensible assumption as the method should be open to the possibility of a target anywhere in the image.

$A$ is a constant, that can be seen as a user parameter. If $A$ is chosen large, all $\delta_{i}$ are allowed to vary with less restrictions.

\section{A. Minimization Under Constraints}

To minimize $J$ under the constraints (15) and (16), find the stationary points of the Lagrangian (see [16] for details):

$$
\begin{aligned}
J_{L} & =J+\mu_{1}\left(\frac{1}{M N} \sum_{i} \frac{1}{\delta_{i}^{2}}-\lambda_{0}\right) \\
& +\mu_{2}\left(\frac{1}{M N} \sum_{i} \delta_{i}^{2}-A\right),
\end{aligned}
$$

where $\mu_{1}$ and $\mu_{2}$ are Lagrange multipliers. Because $J_{L}$ is dependent on both $\mathbf{o}$ and $\mathbf{C}$, the stationary point is sought after by alternatively keeping one of these fixed, while finding the stationary point of the other. Differentiation of (17) with respect to $\mathbf{o}$ yields the same expression for $\hat{\mathbf{o}}$ as in (7).

In the second optimization step, the ô that was found in the previous step is fixed. Finding the stationary point of $J_{L}$ with respect to $\mathbf{C}$ is more complicated. We wish to equate the following derivatives to zero:

$$
\begin{aligned}
\left.\frac{d J_{L}}{d \delta_{i}^{2}}\right|_{\mathbf{o}=\hat{\mathbf{o}}}=\frac{d J_{L}}{d \delta_{i}^{2}} & {\left[\frac{1}{2} \sum_{i} \frac{\hat{o}_{i}^{2}}{\delta_{i}^{2}}+\mu_{1}\left(\frac{1}{M N} \sum_{i} \frac{1}{\delta_{i}^{2}}-\lambda_{0}\right)\right.} \\
+ & \left.\mu_{2}\left(\frac{1}{M N} \sum_{i} \delta_{i}^{2}-A\right)\right] \\
& =\frac{1}{2}\left(-\frac{\hat{o}_{i}^{2}}{\delta_{i}^{4}}\right)+\mu_{1}\left(-\frac{1}{\delta_{i}^{4}}\right)+\mu_{2}(1),
\end{aligned}
$$

for all $\delta_{i}^{2}, i=1 \ldots M N$. This yields:

$$
\delta_{i}^{2}=\sqrt{\frac{\mu_{1}+\frac{M N}{2} \hat{o}_{i}^{2}}{\mu_{2}}},
$$

and inserting this into the constraints (15) and (16) results in: 

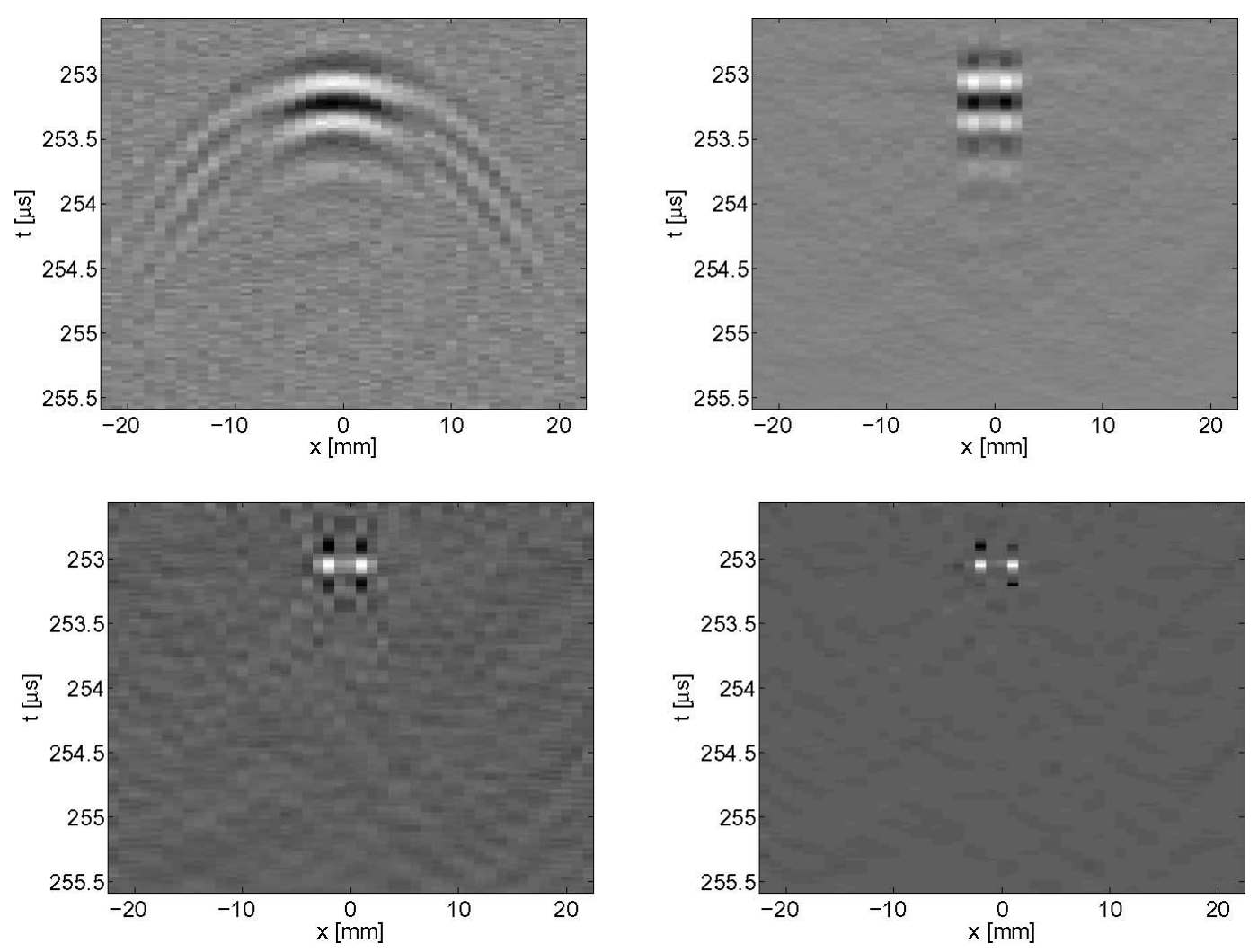

Fig. 3. Simulation results for $4 \mathrm{~mm}$ transducer. Original B-scan (top left), standard SAFT (top right), standard ESAFT (bottom left), iterative ESAFT (bottom right).

$$
\begin{aligned}
& \lambda_{0}=\sum_{i} \sqrt{\frac{\mu_{1}+\frac{M N}{2} \hat{o}_{i}^{2}}{\mu_{2}}}, \\
& A=\sum_{i} \sqrt{\frac{\mu_{2}}{\mu_{1}+\frac{M N}{2} \hat{o}_{i}^{2}}} .
\end{aligned}
$$

This is a system of nonlinear equations with two unknowns that cannot be solved analytically; it must be solved numerically. When $\mu_{1,2}$ has been found, all $\delta_{i}$ can be calculated readily from (19). These steps now can be iterated until $J_{L}$ in (17) converges.

Note that if $\mu_{1}$ is close to zero, which may occur when $A$ is large, then the expression in (19) reduces to $\delta_{i}^{2} \approx \alpha \hat{o}_{i}$; the estimated variances for the next step will simply be the estimated image from the recent step, scaled by some constant $\alpha$.

\section{Results}

Experiments were performed to evaluate the performance of the proposed method. A linear rectangular array, geometrically focused in elevation, was used in the experiments as a variable sized transducer. The transducer size was changed by bridging a varying number of elements, for example 4 or 16 . The measurements were performed in immersion with targets located in the array's geometrical focus in the setup shown in Fig. 7. Simulations of

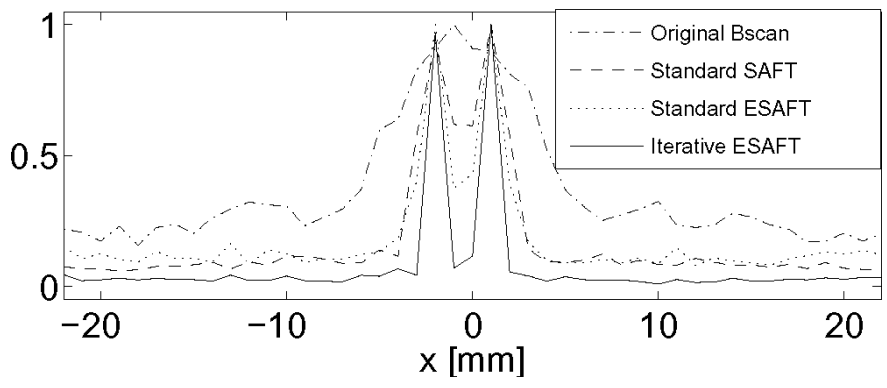

Fig. 4. Simulation results for $4 \mathrm{~mm}$ transducer. Profile plot.

the experimental setup were performed first, and the simulation results were used to compare the iterated ESAFT algorithms with an ordinary time-domain delay and sum SAFT. A fixed size synthetic aperture consisting of 35 elements without apodization was used in the delay-and-sum SAFT. Linear interpolation between discrete samples was also used. Below simulated results are presented, followed by results from measurements.

\section{A. Simulations}

Simulations of the imaging setup used in the measurements presented in the next section were performed using the Discrete REpresentation Array Modelling (DREAM) toolbox [17]. Referring to the geometry described in Fig. 1, the transducer was modeled as a strip, infinitely thin in the $y$-direction and with a variable, finite length in the $x$ direction. The ROI was located entirely in the $x z$-plane, 

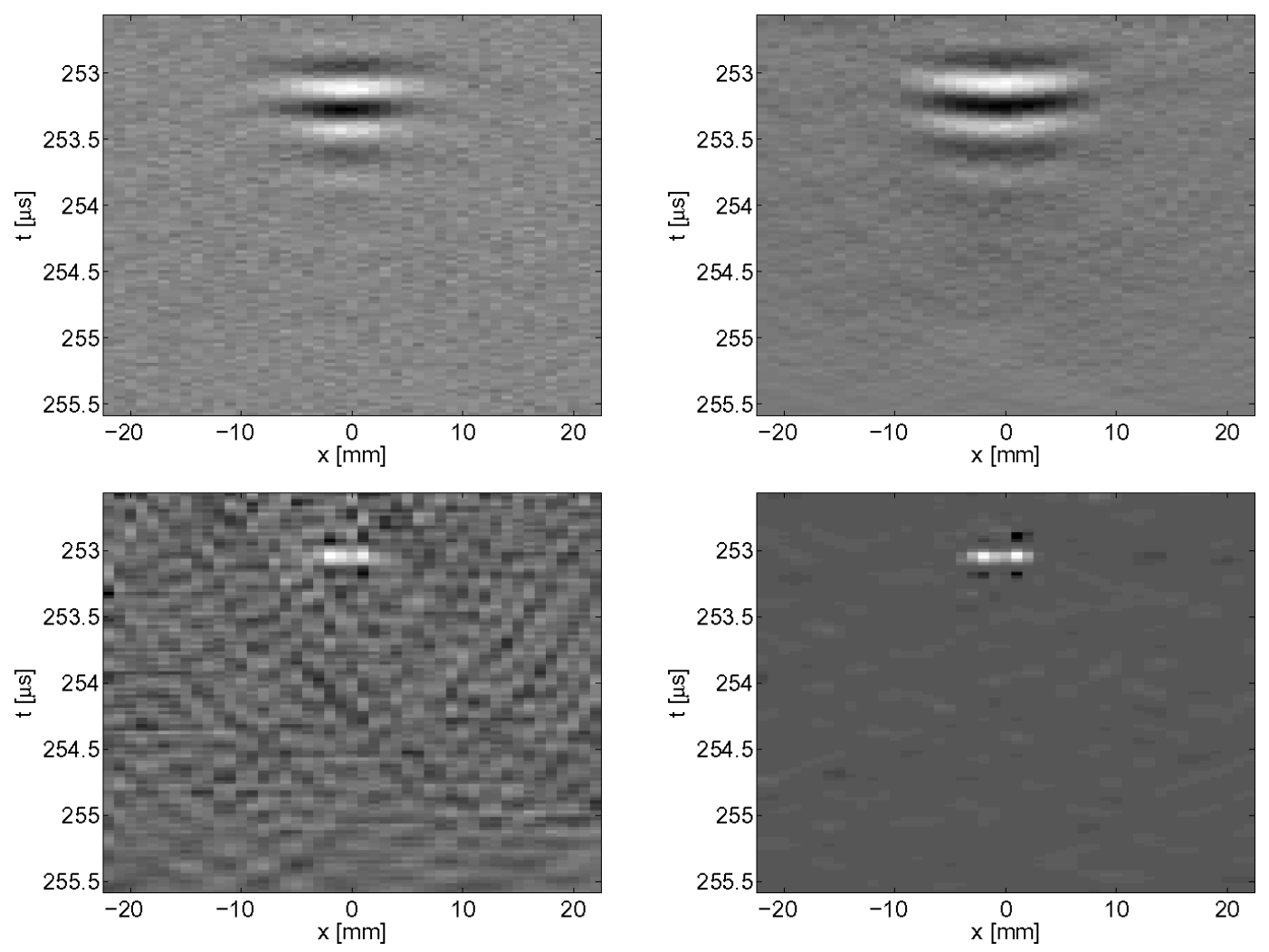

Fig. 5. Simulation results for $16 \mathrm{~mm}$ transducer. Original B-scan (top left), standard SAFT (top right), standard ESAFT (bottom left), iterative ESAFT (bottom right).

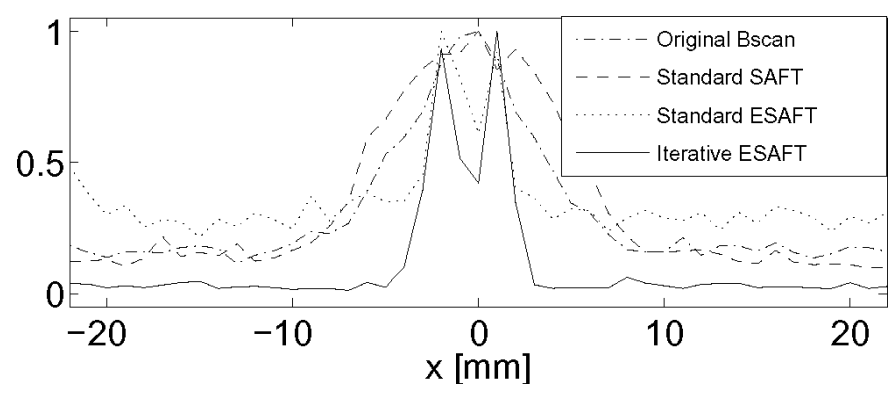

Fig. 6. Simulation results for $16 \mathrm{~mm}$ transducer. Profile plot.

and neither transducer nor targets extended beyond this plane.

In the real measurements, described in more detail in Section IV, the transducer was focused geometrically in the $y$-direction, so that the targets were in the focal zone at the symmetry plane. The spatial impulse responses for observation points close to the targets then should be identical for the (simulated) line source and the (real) focused transducer.

The simulations are made for a broadband transducer with a center frequency of $3 \mathrm{MHz}$ with a double path impulse response shown in Fig. 2. The electrical impulse response used in the simulations was measured from the real array used in the measurements presented in Section IV. A short, rectangular pulse was used as excitation pulse. The impulse response also includes the effects of the excitation pulse.

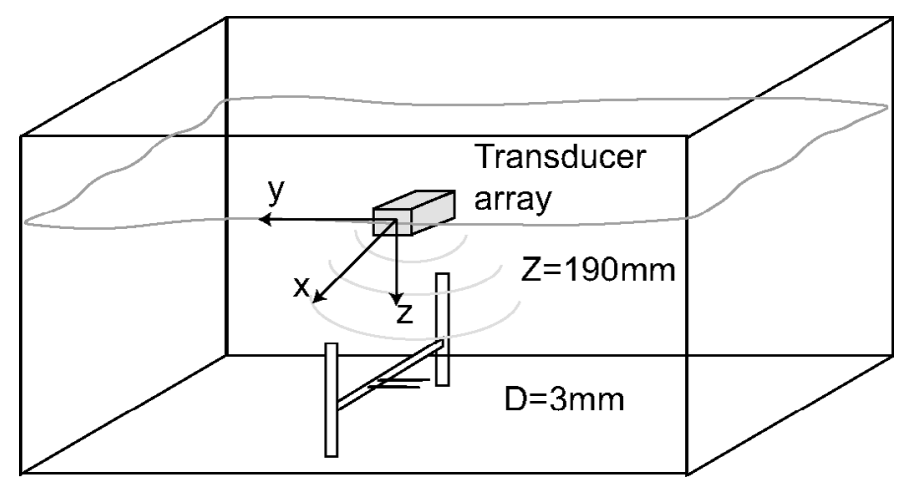

Fig. 7. Measurement setup. Two closely spaced wires in water.

Two closely spaced point targets, located in water at a distance of $190 \mathrm{~mm}$ from the transducer, corresponding to the real array's focal distance, and separated by $3 \mathrm{~mm}$ were simulated. The transducer aperture size was set to either 4 or $16 \mathrm{~mm}$, and the spatial sampling distance was $1 \mathrm{~mm}$, which is equal to $2 \lambda$ for the transducer's center frequency $3 \mathrm{MHz}$ in water. White Gaussian noise was added to the data after the convolution with electrical impulse response, to obtain the SNR of $10 \mathrm{~dB}$. The simulated radio frequency (RF) signals were sampled at $40 \mathrm{MHz}$. The choice of point targets was motivated by two purposes: providing a good measurement setup for evaluating spatial resolution, and creating a reasonably realistic situation for NDE purposes in which scatterers often are few and small. 

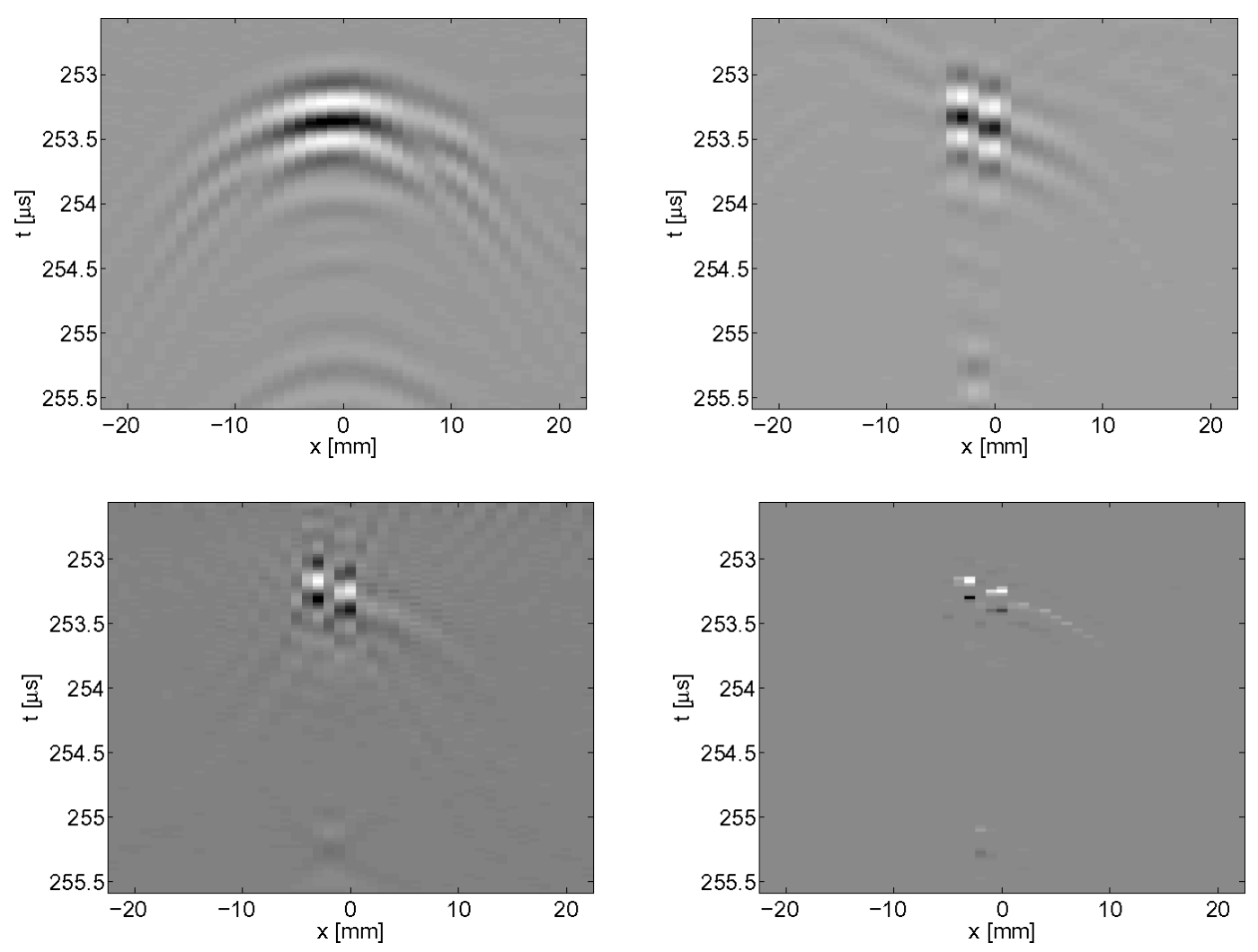

Fig. 8. Measured results for $4 \mathrm{~mm}$ transducer. Original B-scan (top left), standard SAFT (top right), standard ESAFT (bottom left), iterative ESAFT (bottom right).

In Figs. 3 to 6, the results of the simulations are shown. The intensity plots show B-scans consisting of aggregated RF-signals acquired at all transducer positions. Note that the term B-scan, which is used in medical applications for aggregated signal envelopes, refers in NDE to aggregated RF-signals. To simplify comparisons between different algorithms, data have been normalized to have its respective maximum amplitude set to unity. The profile plots were generated by taking the maximum from each column of the image. All reconstructed images are made with $45 \times 121$ image elements (pixels) corresponding to a ROI of $45 \times 4.5 \mathrm{~mm}$.

From the results presented in Figs. 3 to 6 can be seen that both ESAFT and iterative ESAFT can distinctly separate the two targets, but the iterative approach is clearly superior in suppressing the noise. Interestingly, no side lobes appear in the iterative ESAFT profiles, the background noise level is rather constant outside the peaks.

As mentioned before, in order to obtain the spatial resolution sufficient to separate the targets, a small $\lambda_{0}$ must be chosen in (11) for the single-step ESAFT algorithm. This choice yields small or no damping of the noise present in the image. The iterative algorithm starts with larger value of $\lambda_{0}$, and updates it in subsequent steps.

\section{B. Measurements}

Ultrasonic data was collected using an array system with a $3 \mathrm{MHz}$ focused array from Imasonic. The array consists of 64 rectangular elements spaced with $1 \mathrm{~mm}$. The

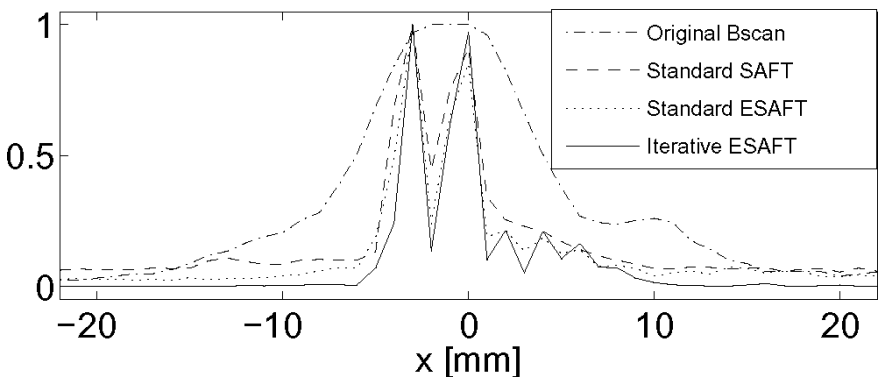

Fig. 9. Measured results for $4 \mathrm{~mm}$ transducer. Profile plot.

elements that are $0.95 \mathrm{~mm}$ wide strips can be bridged easily to form larger apertures. If all elements in an aperture are fired simultaneously, it acts like a rectangular source of a size determined by the number of active elements. Two $0.3 \mathrm{~mm}$ thick steel wires, separated by $3 \mathrm{~mm}$ and submersed in water, were used as targets. The number of single-array elements used in the experiments, and thus the approximate transducer width in $\mathrm{mm}$, were, respectively, $1,2,4,8,12$, and 16 . The distance between the targets and the aperture was constant and equal $z=190 \mathrm{~mm}$ as shown in Fig. 7. The array was geometrically focused at this distance in the $y$ (elevation) direction. B-scans were gathered along the $\mathrm{x}$-axis, perpendicular to the wires using a spatial sampling distance of $1 \mathrm{~mm}$. The RF signals were acquired with a sampling rate of $40 \mathrm{MHz}$ and an 8 bit analog-to-digital converter. Results obtained for the $4 \mathrm{~mm}$ and $16 \mathrm{~mm}$ aperture are presented in Figs. 8 to 11. 

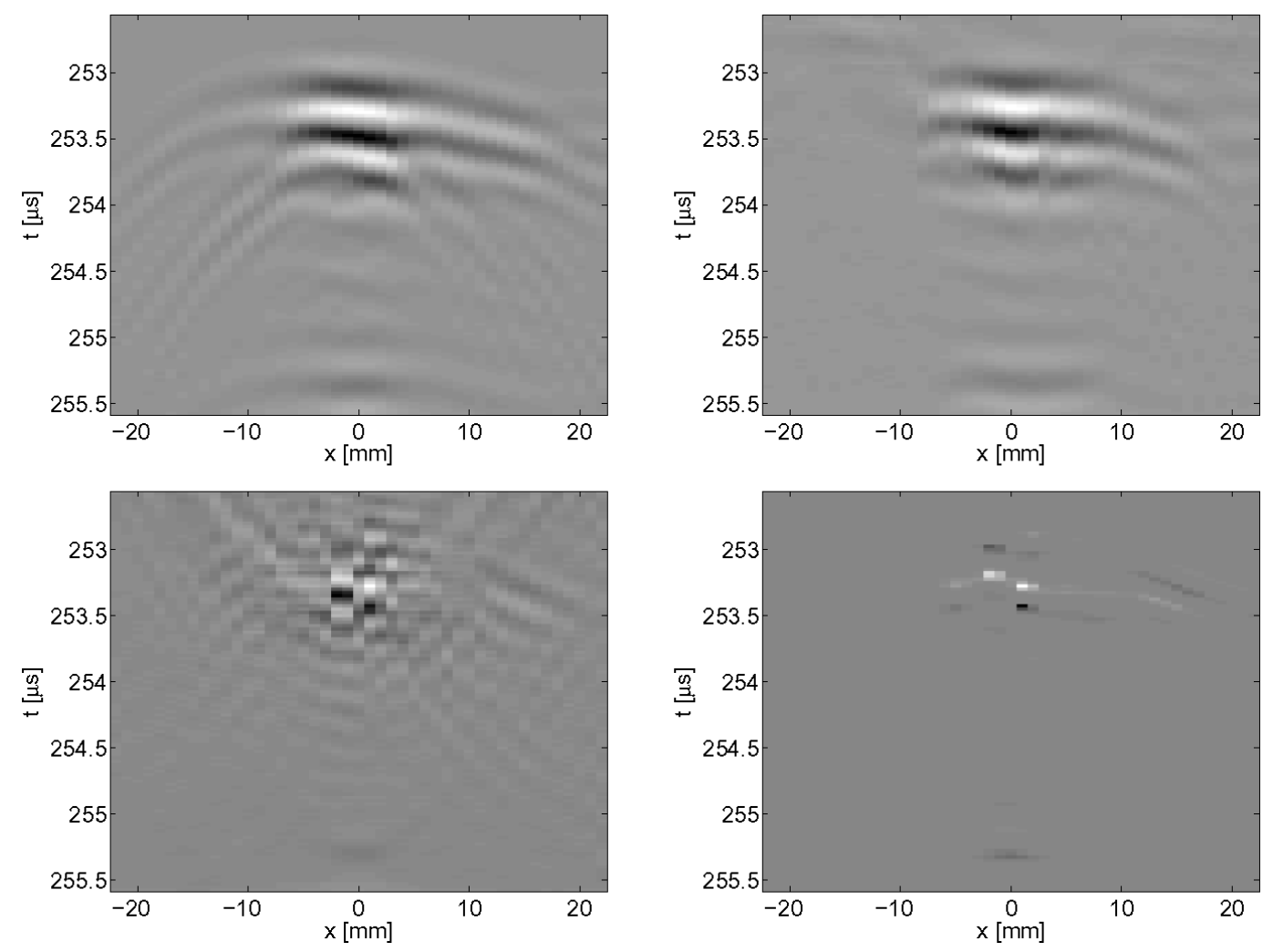

Fig. 10. Measured results for $16 \mathrm{~mm}$ transducer. Original B-scan (top left), standard SAFT (top right), standard ESAFT (bottom left), iterative ESAFT (bottom right).

For a narrow, rectangular transducer of length $L$, the far field approximation is valid for distances beyond $r>$ $L^{2} / 4 \lambda$ [18]. Although the ESAFT algorithm does not use the far field approximation in any step, due to practical reasons (an available array), for all aperture sizes used in the measurements, the targets were located in the transducer's far field. However, ESAFT is capable of compensating transducer diffraction effects in the near field if the respective SIRs are known.

The results from measured data are similar to the simulated ones. Some reduction of performance can be observed, but it was expected, as the model of the transducer is not perfect. The iterative ESAFT algorithm performs as well as, or better than, the original EASFT method with regard to the resolution, and additionally it is capable of suppressing noise to a greater extent.

The evolution steps of the iterative approach is illustrated in Figs. 12 and 13 showing evolution of B-scan profiles in successive iterations for the $8 \mathrm{~mm}$ aperture. Note that the larger the aperture, the more its SIR deviates from the ideal SIR of a point like transducer. In consequence, when the ultrasonic measurement performed using a large aperture is processed using an ordinary SAFT, a satisfactory spatial resolution is difficult to achieve [13]. In this context, $8 \mathrm{~mm}$ aperture of a $3 \mathrm{MHz}$ transducer in water is fairly large for targets at a distance $190 \mathrm{~mm}$.

From the plots in Fig. 12 can be seen that, after the first iteration of the proposed algorithm, the two wires have not been separated, but after further steps they can be distinguished clearly.

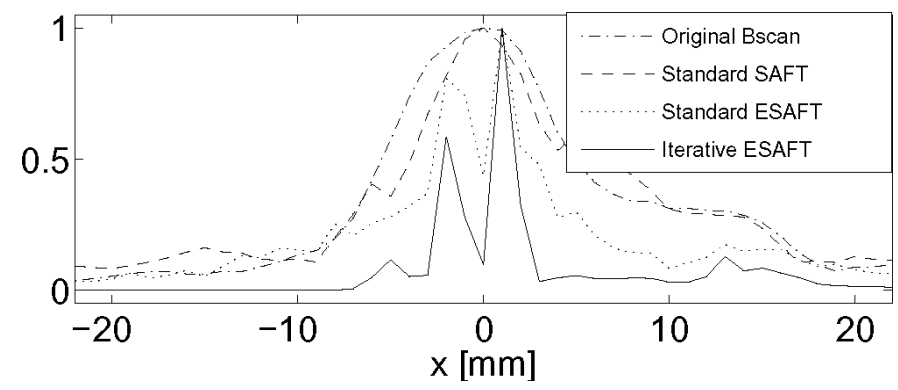

Fig. 11. Measured results for $16 \mathrm{~mm}$ transducer. Profile plot.

In the example presented above, the initial matrix $\mathbf{C}_{\mathbf{0}}$ in the step 1 of our algorithm was set to a relative high value $\lambda_{0}=1 e^{-4}$. For lower start values (for example, $\lambda_{0}=$ $\left.1 e^{-6}\right)$, the wires could be distinguished after processing using the original single-step ESAFT approach, see Fig. 13. Note, however, that this is achieved at the price of a higher noise floor and less distinct peaks corresponding to the wires comparing to the iterative SAFT result in Fig. 12(i). This is clear evidence that the iterative approach is more successful in suppressing the background noise than the single-step ESAFT.

\section{Impact of Aperture Size}

Performance of SAI algorithms generally decreases with increasing apertures; see [5] for more details on impact of transducer size on imaging. This is especially true for SAFT but also for ESAFT. Indeed, the spatial impulse 


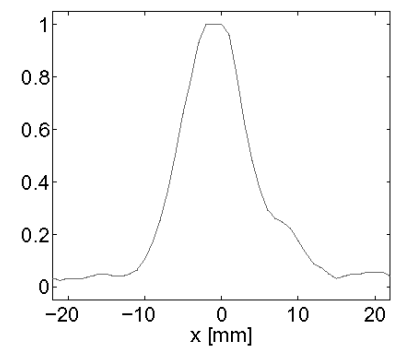

(a) Original data.

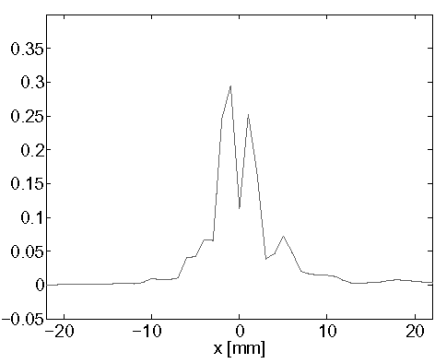

(e) Iteration 4 .

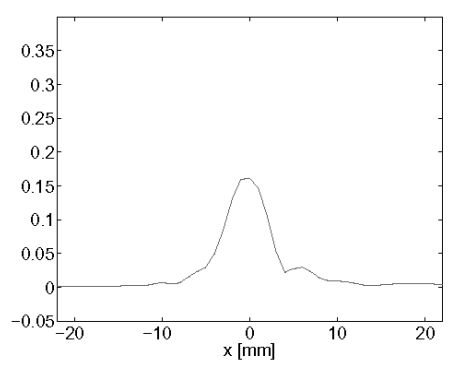

(b) Iteration 1 .

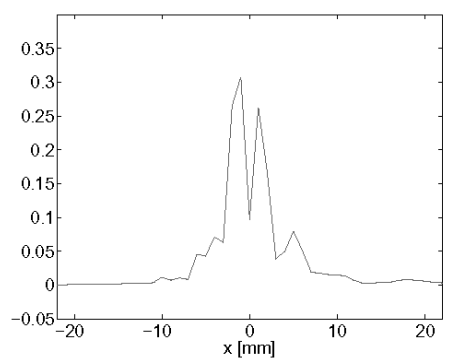

(f) Iteration 5 .

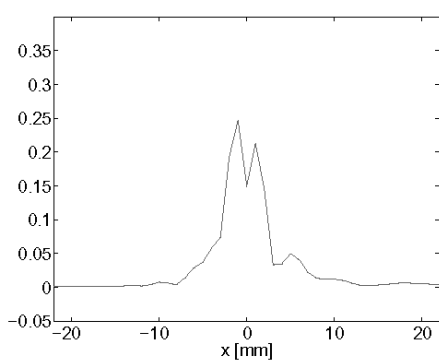

(c) Iteration 2 .

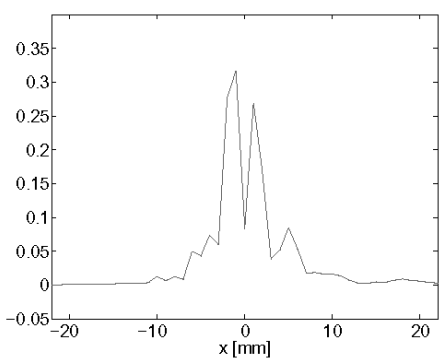

(g) Iteration 6 .

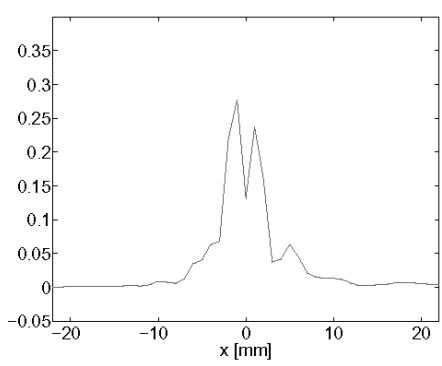

(d) Iteration 3 .

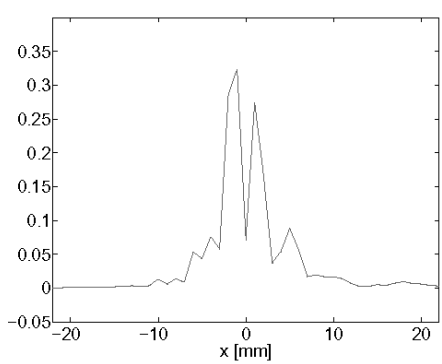

(h) Iteration 7 .

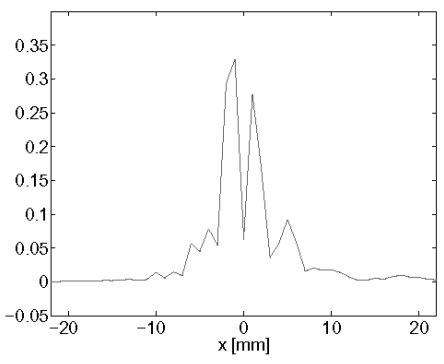

(i) Iteration 8 .

Fig. 12. Profile plots obtained for $8 \mathrm{~mm}$ transducer, $\lambda_{0}=1 e^{-4}$. Note gradual resolution enhancement, after the first iteration the two wires cannot be distinguished.

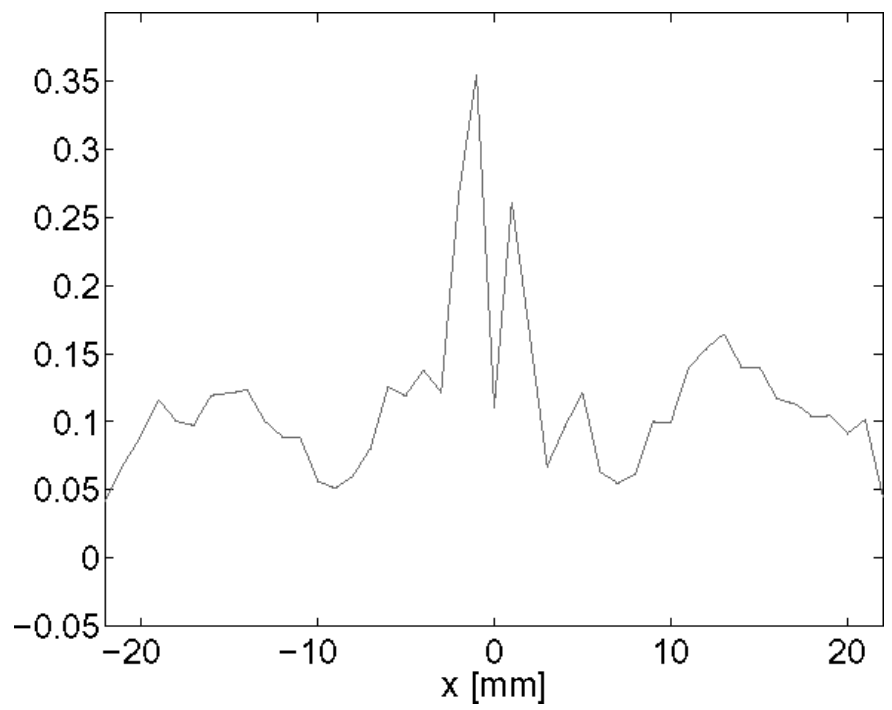

Fig. 13. The result obtained for $8 \mathrm{~mm}$ transducer, without the iterative approach for $\lambda_{0}=1 e^{-6}$.

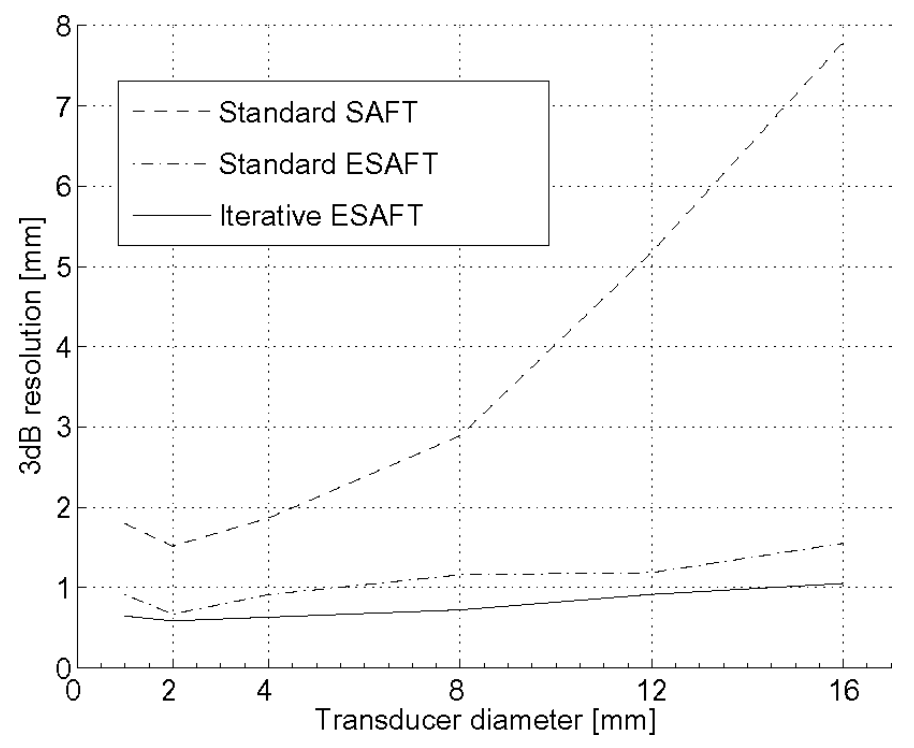

Fig. 14. $3 \mathrm{~dB}$ resolution as a function of aperture size. 
response of the finite-sized transducer acts as a low-pass filter for the received signal and because the SIR length increases with the size of the aperture, the higher frequencies present in the target response are more and more attenuated. This effect is especially well pronounced for larger angles of the incident beams. Below, a comparison of the $3 \mathrm{~dB}$ lateral resolution for SAFT, ESAFT, and the iterative ESAFT is presented. The data was acquired using the same setup as described in Section IV, but only a single wire was used as target.

It can be seen from Fig. 14 that the iterative ESAFT approach offers clear improvement over the standard ESAFT algorithm, and that both are superior to SAFT. The resolution of the ESAFT methods decreases much slower with increasing aperture size, as they maintain reasonable accuracy for apertures as large as $16 \mathrm{~mm}$.

\section{Conclusions}

An iterative version of ESAFT algorithm was proposed in this paper and tested using both simulated and real ultrasonic data. The algorithm uses a sequential optimization scheme in which the parameters found in the previous step is used for minimizing the reconstruction error in the present step. It has been shown that the algorithm yields an improved lateral resolution comparing with the ordinary ESAFT.

It also has been shown that the iterative approach is relatively insensitive to the initial choice of the user parameters, which is an important practical advantage. Performance of the original ESAFT method, which is essentially a single iteration of the iterative version presented here, depends heavily on the initial estimates of $\sigma_{o}^{2}$ and $\sigma_{e}^{2}$ in (10). The iterative approach presented in this work minimizes the error criterion (17) until it converges, which makes it less dependent on the initial choice of parameters. Thus, it is more robust and is more suited for practical NDE applications, such as automated defect detection/classification than the original ESAFT.

\section{REFERENCES}

[1] J. A. Saydel, Ultrasonic Synthetic-Aperture Focusing in NDT. R. S. Sharpe, Ed. New York: Academic, 1982.

[2] A. A. Winder, "Sonar system technology," IEEE Trans. Sonics Ultrason., vol. SU-22, no. 5, pp. 291-332, 1975.

[3] M. I. Skolnik, Introduction to Radar Systems. New York: McGraw-Hill, 1980.

[4] L. J. Cutrona, "Comparison of sonar system performance achievable using synthetic-aperture technique with the performance achievable by more conventional means," J. Acoust. Soc. Amer., vol. 58, no. 2, pp. 336-348, Aug. 1975.

[5] R. N. Thomson, "Transverse and longitudinal resolution of the synthetic aperture focusing technique," Ultrasonics, vol. 22, pp. 9-15, Jan. 1984.

[6] C. Passman and H. Ermert, "A $100 \mathrm{MHz}$ ultrasound imaging system for dermatologic and ophthalmologic diagnostics," IEEE Trans. Ultrason., Ferroelect., Freq. Contr., vol. 43, no. 4, pp. 545-552, July 1996.

[7] C. H. Frazier and W. D. O'Brien, "Synthetic aperture techniques with a virtual source element," IEEE Trans. Ultrason., Ferroelect., Freq. Contr., vol. 45, pp. 196-207, Jan. 1998.

[8] M-H. Bae and M-K. Jeong, "A study of synthetic-aperture imaging with virtual source elements in B-mode ultrasound imaging systems," IEEE Trans. Ultrason., Ferroelect., Freq. Contr., vol. 47, pp. 1510-1519, Nov. 2000.

[9] S. I. Nikolov, "Synthetic aperture tissue and flow ultrasound imaging," Ph.D. dissertation, Technical University of Denmark, Lyngby, Denmark, 2001.

[10] F. Lingvall, T. Olofsson, and T. Stepinski, "Synthetic aperture imaging using sources with finite aperture: Deconvolution of the spatial impulse response," J. Acoust. Soc. Amer., vol. 114, no. 1, pp. 225-234, July 2003.

[11] T. Stepinski, E. Wennerström, F. Lingvall, and P. Wu, "Inspection of copper canisters for spent nuclear fuel by means of ultrasound-NDE of friction stir welds, nonlinear acoustics, ultrasonic imaging," Technical Report TR-04-03, Swedish Nuclear Management Co., Stockholm, Sweden, 2003.

[12] F. Lingvall, "A method of improving ovrtall resolution in ultrasonic array imaging using spatio-temporal deconvolution," $U l$ trasonics, vol. 42, pp. 961-968, 2004.

[13] F. Lingvall, "Time domain reconstruction methods for ultrasonic array imaging," Ph.D. dissertation, Uppsala University, Signals and Systems, Uppsala, Sweden, 2004.

[14] T. Olofsson, "Semi-sparse deconvolution robust to uncertainities in the impulse responses," Ultrasonics, vol. 42, pp. 969-975, 2003.

[15] H. Lasota and R. Salamon, "Application of time-space impulse responses to calculations of acoustic field in imaging systems," Acoust. Imaging, vol. 10, pp. 493-512, 1980.

[16] R. Fletcher, Practical Methods of Optimization. 2nd ed. New York: Wiley, 1987.

[17] B. Piwakowski and K. Sbai, "A new approach to calculate the field radiated from arbitrarily structured transducer arrays," IEEE Trans. Ultrason., Ferroelect., Freq. Contr., vol. 46, pp. 422-440, 1999.

[18] L. E. Kinsler, A. R. Frey, A. B. Coppens, and J. V. Sanders, Fundamentals of Acoustics. 4th ed. New York: Wiley, 2000. 